\title{
Reliability of haemodynamic monitoring using wave form analysis in major peripheral vascular surgery
}

\author{
Lauretta M.P., Courtois N., Illingworth J., Gautama S., Sidhu V., Platt M. \\ *Clinical fellow, anaesthetic department. St. Mary's Hospital, London. Imperial College Healthcare NHS trust. \\ ** Consultant, anaesthetic department. St. Mary's Hospital, London. Imperial College Healthcare NHS trust•
}

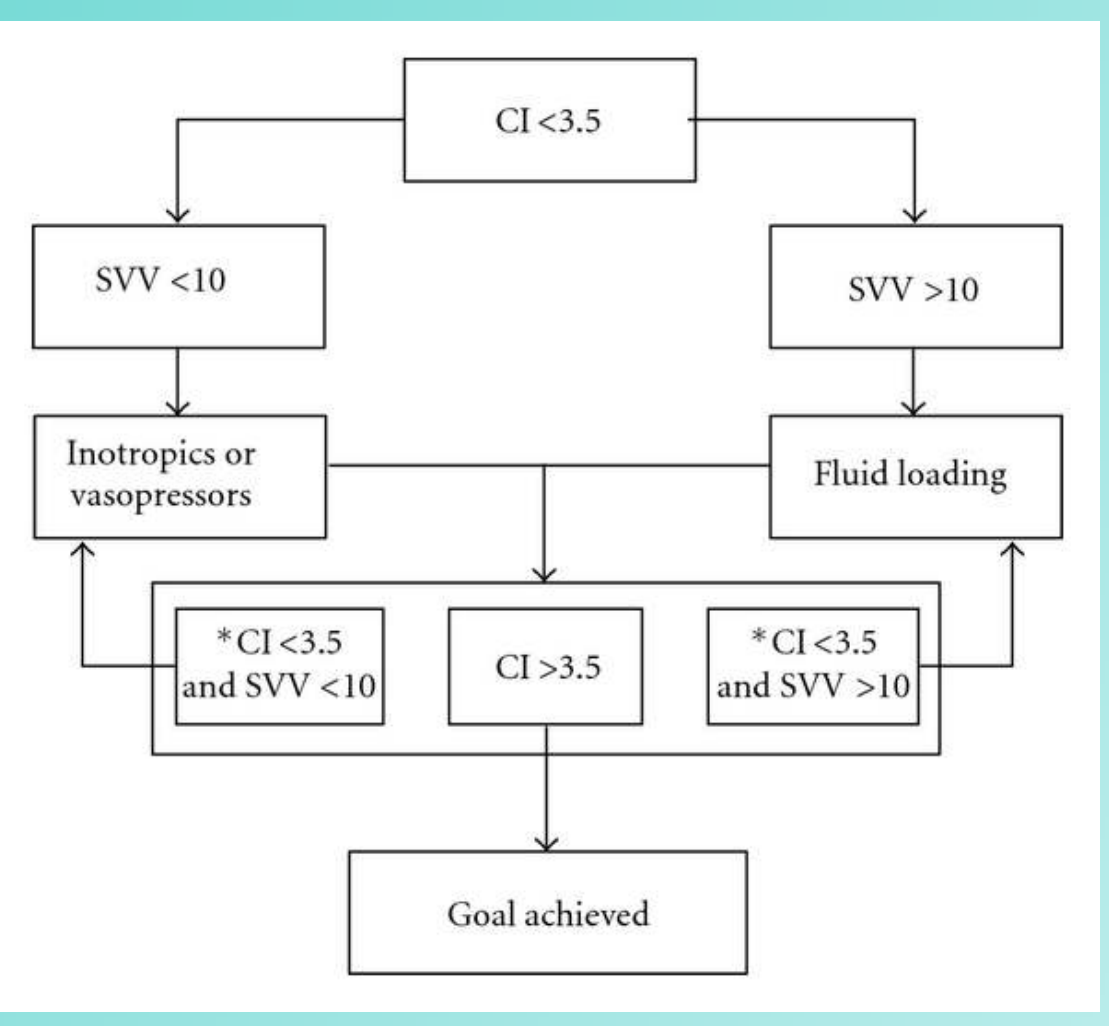

\section{Background:}

Recent guidelines from UK health service suggest that haemodynamic monitoring should be used in high risk patients undergoing major surgery to reduce mortality, morbidity and reduce costs. However, in vascular surgery, haemodynamic monitoring is challenging, and the expensive and invasive pulmonary artery catheter (PAC) remains the gold standard for cardiac output (CO) monitoring. Aim of this study is to verify if the less invasive Flo/Trac Vigileo, based on arterial waveform analysis, and patient's age, sex, height, weight, correlates with the expected physiological change of the arterial clamping/unclamping in vascular surgery.

\section{Materials and Methods:}

The FloTrac/Vigileo system estimates $\mathrm{CO}$ by multiplying the HR by the SV. This stroke volume is averaged and displayed every $20 \mathrm{~s}$. We used version 1.9 of the software.

Six patients undergoing elective femoral-popliteal bypass between October 2016 and December 2016 were enrolled. Exclusion criteria were: spontaneous breathing, atrial fibrillation, moderate or severe valvular disease, intracardiac shunts, ascites.

Cardiac Index (Cl), Cardiac Output (CO), Stoke Volume (SV), Stroke Volume Variation (SVV), Stroke Volume Index (SVI), Systemic Vascular Resistance (SVR) and Systemic Vascular Resistance Index (SVRI) were recorded at this timing: at incision, arterial clamping, every $10 \mathrm{~min}$ after clamping/unclamping.

\section{Results:}

The Cl, SVI, and SVRI were used for the analysis to eliminate the effect of physical size.

Statistical differences were assessed using a paired T-test. $\mathrm{P}$ values < 0.05 were considered statistically significant. In all our cases application of clamping caused a sudden increase in SVRI (I5\%) and BP, due to the sudden impedance to arterial flow and increased LV afterload ( $p<$ 0.05). In one case we recorded a significant decrease in $\mathrm{Cl}(14 \%)$ and SVI (I5\%) as a result of myocardial dysfunction.

Removal of arterial clamping resulted in a sudden decrease in SVRI $(16 \%)$ and BP, due to reperfusion. SVI increased as a result of venodilation and decreased preload $(14 \%),(p<0.05)$.

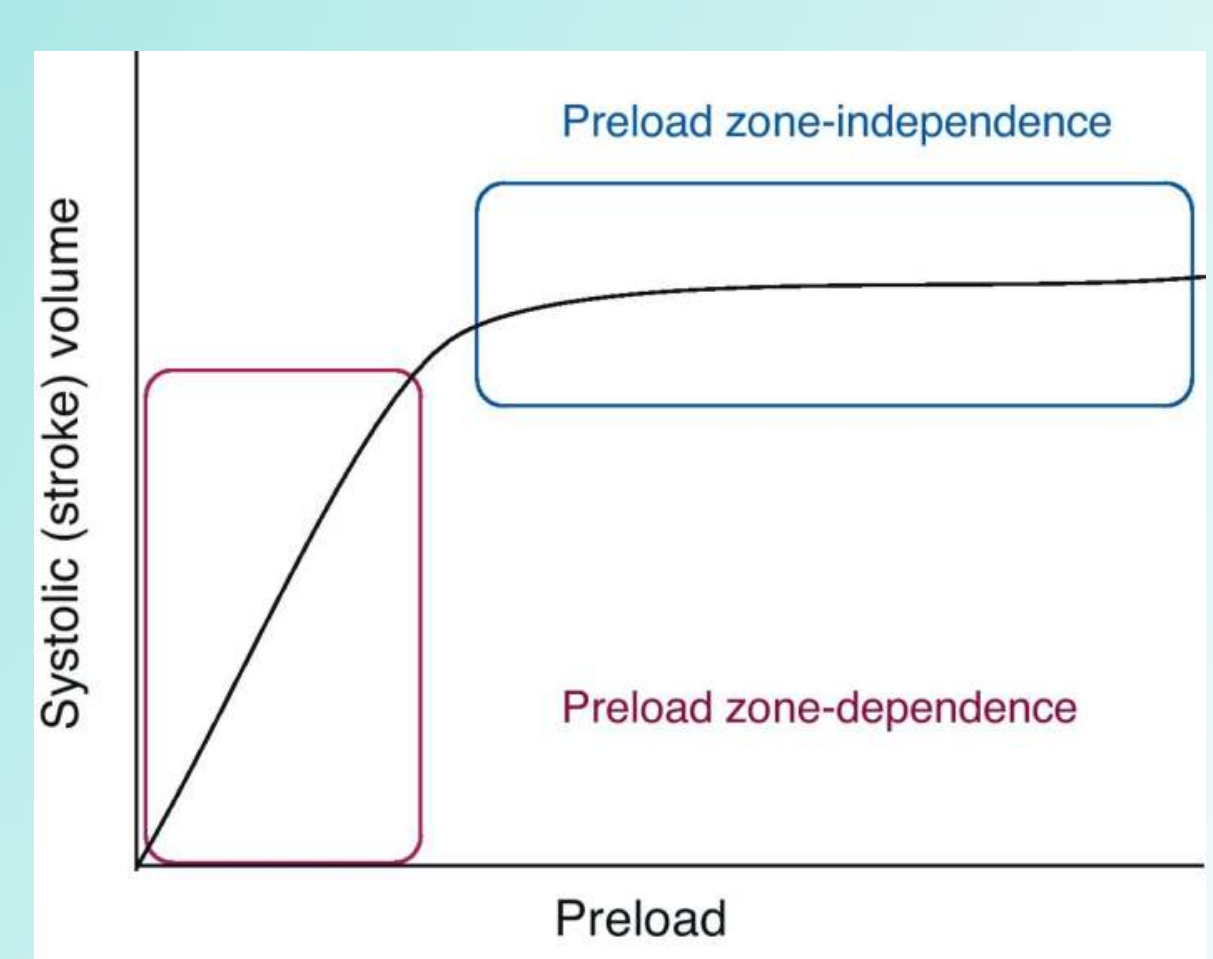

\section{Conclusion:}

This study, even if performed without using PAC for comparison, have shown a good correlation between arterial wave form analysis derived parameters and the expected physiological changes of arterial clamping and unclamping. These semi invasive devices may be promising among high risk patients undergoing peripheral vascular surgery to monitor and optimise $\mathrm{CO}$. This hypothesis is still under investigation.

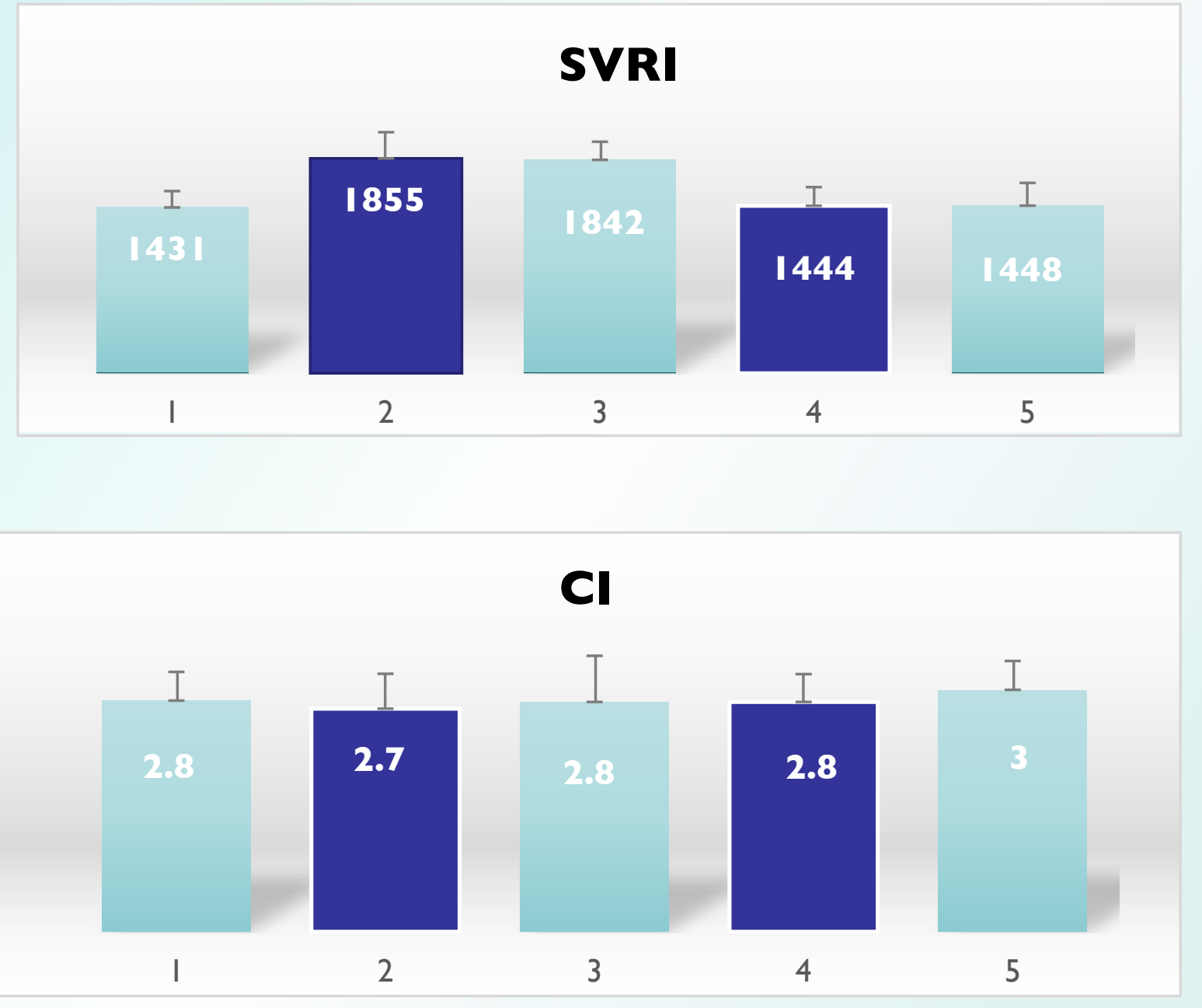

\section{Sources:}

